\title{
Biomarker potential of IncRNA GNAS-AS1 in osteosarcoma prognosis and effect on cellular function
}

\author{
Zhanhu Mi ${ }^{1}$, Yanyun Dong ${ }^{2}$, Zhibiao Wang $^{3}$ and Peng Ye $\mathrm{i}^{1^{*}}$
}

\begin{abstract}
Background: Osteosarcoma (OS) is a type of bone cancer that occurs in children and adolescents at a rate of 5\%. The purpose of this study is to explore the IncRNA GNAS-AS1 expression profile, prognosis significance in OS, and biological effect on OS cell function.

Methods: One hundred eight pairs of tissues were collected, and OS cell lines were purchased. IncRNA GNAS-AS1 expression in these tissues and cells were analyzed by qRT-PCR. Clinical data were analyzed using chi-square tests, Kaplan-Meier curves (log-rank test), and Cox regression. CCK-8 and transwell assay were conducted to analyze the effect of IncRNA GNAS-AS1 on cell proliferation, invasion, and migration. The downstream miRNA was presumed.

Results: The expression of IncRNA GNAS-AS1 was significantly increased in OS cells and tissues, and related to Enneking staging and distant metastasis. Patients with high IncRNA GNAS-AS1 expression represented shorter overall survival and was an independent prognostic predictor of OS. LncRNA GNAS-AS1 knockdown inhibited cell proliferation, migration, and invasion by regulated miR-490-3p partly at least.
\end{abstract}

Conclusions: LncRNA GNAS-AS1 can be used as a prognostic indicator and its inhibition suppress the development of OS, suggesting its value as novel therapeutic strategies in OS.

Keywords: Osteosarcoma, IncRNA GNAS-AS1, Prognosis, Proliferation, Migration, Invasion

\section{Introduction}

Characterized by mineralizing of osteoid with multinucleated cells, osteosarcoma (OS) is the most common tumor in bone tissues among children and adolescents $[1,2]$. The improvement in survival of osteosarcoma has been notable on some levels, for instance, 3D tumor simulation which creates precise anatomical visualization has added benefits for radiologists, and facilitated diagnoses and surgical planning [3, 4]. Despite treatment of localized OS adhering to accepted standards, OS recurs in $30 \%$ to $40 \%$ of patients within $2-3$ years of initial

\footnotetext{
* Correspondence: yepeng8905@163.com

'Department of Trauma Orthopedics, General Hospital of Ningxia Medical University, 804 South Shengli Street, Yinchuan, Ningxia 750004, People's Republic of China

Full list of author information is available at the end of the article
}

treatment. Recurrence usually carries a poor prognosis, and survival after recurrence is greatly reduced [5]. Till now, the biological characteristic of OS has been well understood, but the molecular mechanisms of OS origination, metastasis, or chemoresistance remain fuzzy in large regions [6]. As defined by translational medicine, the advances in basic science research would benefit the effective translation into new approaches for prevention, diagnosis, and treatment of disease for improving health [7]. Thus, new candidate molecules as prognostic biomarkers are expected to be developed, which may profoundly improve clinical outcomes for patients with OS.

Encouragingly, some long non-coding RNAs (lncRNAs) have been reported to serve as predictive, prognostic, and therapeutic biomarkers of human 
cancers recently, since they are usually aberrantly expressed in disease tissues and promote or inhibit tumor development, progression, and metastasis [8-11]. Altering from the perception as 'junk' transcriptional products, lncRNAs have been recognized to play cellfunction regulatory roles and mediate cellular processes including transcription, post-transcriptional modifications, and signal transduction [12]. In OS, lncRNAs participate in cell invasion, migration, proliferation, apoptosis, and drug resistance [13-15], and offer new means for prognosis and therapy of OS [16]. LncRNA GNAS-AS1 was reported to facilitate nasopharyngeal carcinoma, ER+ breast cancer, and lung cancer cell progression [17-19]. Though LncRNA GNAS-AS1 was screened as dysregulated lncRNA in OS [20], but its role in OS has not been discussed.

In this study, the expression level of IncRNA GNASAS1 in OS would be determined. Based on the aberrant expression level, the potential value as prognosis predictor was accessed. The effect of lncRNA GNAS-AS1 interference on OS cellular function was analyzed, and the underly mechanism was preliminarily investigated.

\section{Materials and methods}

\section{Patients and tumor samples}

This study included frozen biopsy samples of 108 patients (age range 8-48 years, mean 16.7 years) who were diagnosed OS at General Hospital of Ningxia Medical University from March 2008 to December 2015. They were followed up by telephone survey at least every 3 months after completion of surgery for 2 years, and then every 6 months for at least 5 years. Two pathologists reviewed the collected biopsy specimens. This retrospective study was approved by the Institutional Review Board of General Hospital of Ningxia Medical University. Written informed consent was obtained from each participant according to the Declaration of Helsinki.

\section{Cell lines culture and transfection}

Four human OS cell lines (MG-63, Saos-2, SW 1353, U2 OS) and one normal osteoblast cell line, hFOB 1.19 were obtained from the National Collection of Authenticated Cell Cultures (Shanghai, China). MG-63, Saos-2, and SW 1353 cells were maintained in Dulbecco's modified Eagle medium (DMEM, Gibco, cat\# 11965092, USA), U2 OS cells in McCoy's 5A medium (Gibco by Life Technologies, cat\# 16600082), and hFOB 1.19 cells in a mixture of DMEM and Ham F12 medium (1:1 ratio, Gibco). The cell culture environment was in a humid incubator.

The siRNAs (si-GNAS-AS1 and si-control) were delegated to Ribobio (Guangzhou, China) for design and synthesis. The miR-490-3p inhibitor (anti-miR) and corresponding negative control (anti-NC) were also synthesized by Ribobio (Guangzhou, China). Lipofectamine 2000 (Invitrogen, USA) was utilized to complete the transfection and qRT-PCR was utilized to evaluate the transfection efficiency.

\section{RNA isolation and quantitative real-time PCR (qRT-PCR)}

Total RNA was extracted via TRIzol reagent (Ambion by Life Technologies, USA) from the tissues and cell lines. The cDNA for IncRNA GNAS-AS1 was generated using lncRcute lncRNA First-Strand cDNA Synthesis kit (with gDNase) (Tiangen, China) and the quantification was performed by lnRcute lncRNA SYBR Green kit (Tiangen, China). miRcute miRNA First-Strand cDNA Synthesis kit and miRcute Plus miRNA qPCR Kit (SYBR Green; Tiangen, China) were purchased for the quantification of miR-490-3p expression level. GAPDH or U6 was employed as the internal control for quantification of lncRNA GNAS-AS1 and miR-490-3p levels. The relative expression of RNAs was calculated by the $2^{-\Delta \Delta \mathrm{Ct}}$ method.

\section{Cell proliferation assay by cell counting kit-8 (CCK-8)}

Cell proliferation of MG-63 and SW 1353 cells was analyzed with a CCK-8 assay. Cells were incubated in a 96well plate $\left(2 \times 10^{3}\right.$ cells/well $)$ and transfected with the indicated plasmid(s) for $24 \mathrm{~h}$. Subsequently, CCK- 8 solution $(10 \mu \mathrm{L})$ was added to each well every $24 \mathrm{~h}$ for $72 \mathrm{~h}$, and the cells were further incubated for $2 \mathrm{~h}$. The absorbance was measured at $450 \mathrm{~nm}$, and the reference absorbance at $630 \mathrm{~nm}$ with a SpectraMax 190 microplate reader (Molecular Devices, USA). Three repeated experiments were performed.

\section{Transwell assay to determine cell migration and invasion assay}

MG-63 and SW 1353 cells were trypsinized and resuspended with medium without serum. Transwell insert for invasion assay was precoated with Matrigel Matrix (Corning, USA). The cells were plated at a density of $5 \times$ $10^{4}$ cells/well in the upper compartment of transwell insert (Corning, USA). The chemoattractant in the lower compartment was $10 \%$ FBS. After a 24 -h incubation, the uninvaded cells were removed, while the passed cells were fixed with methanol for $1 \mathrm{~h}$ and then stained with $0.1 \%$ crystal violet. Five random fields of vision were taken under the microscope and the stained cells were counted manually.

\section{Target miRNA prediction and luciferase reporter assay} LncBase Predicted v.2 and lncRNASNP2 were used to predict the potential miRNAs targeting lncRNA GNAS-AS1. pLHCX retroviral vector containing wildtype lncRNA GNAS-AS1 (WT- GNAS-AS1) or mutated one (MUT-GNAS-AS1) generated by site- 
directed mutagenesis were purchased from Ribobio (Guangzhou, China). MG-63 cells were co-transfected with wild-type or mutated IncRNA GNAS-AS1 reporter plasmids, and with miR-490-3p mimics or inhibitor or corresponding negative control. Twentyfour hours post-transfection, the luciferase activity was assayed using the 100T Promega E1910 Dual Luciferase Reporter Assay System (Promega, USA) following the manufacturer's instruction.

\section{Statistical analysis}

The data were represented as mean with SD. Student's $t$ test, one-way, or two-way ANOVA was used to calculate the significance of the data. Clinical data were analyzed using chi-square tests, Kaplan-Meier curves (log-rank test), and Cox regression. Chi-square tests were for the access of association between IncRNA GNAS-AS1 and the clinical parameters. Kaplan-Meier curves were plotted to show a direct comparison visually. Next, the statistical significance of each prognostic factor was assessed by comparing likelihood ratios from multivariate Cox analysis. Correlation analysis between
lncRNA GNAS-AS1 and miR-490-3p expression was performed by Pearson's correlation coefficient (Pearson's r) with a two-tailed test. $P<0.05$ was considered significant.

\section{Results}

Upregulation of IncRNA GNAS-AS1 in OS tissues and cell lines

To obtain the expression profile of lncRNA GNAS-AS1 in OS, qRT-PCR was utilized to determine its level in OS tissues, the matched normal tissues, OS cell lines, and normal osteoblast cell line. Compared to the normal tissues, lncRNA GNAS-AS1 was found with an ascendant expression level in OS tissues $(P<0.001$, Fig. $1 \mathrm{~A})$. Similarly, expression of lncRNA GNAS-AS1 in OS cell lines was significantly higher than that in osteoblast cell line hFOB1.19, especially in MG-63 and SW 1353 cell line which would be used in the subsequent test $(P<0.001$, Fig. $1 \mathrm{~B})$. On the contrary, the expression level of miR-490-3p in OS tissues and cell lines was significantly decreased compared with normal ones $(P<0.001$, Fig. $1 \mathrm{C}, \mathrm{D})$.
A

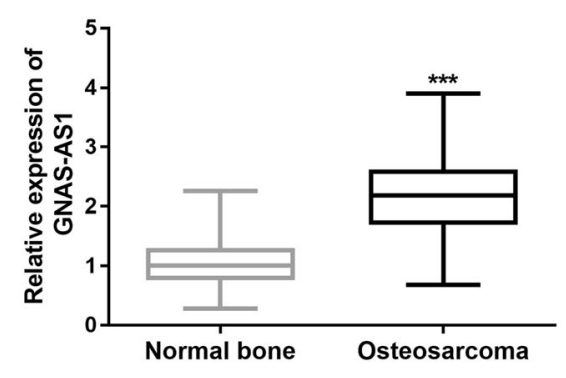

C

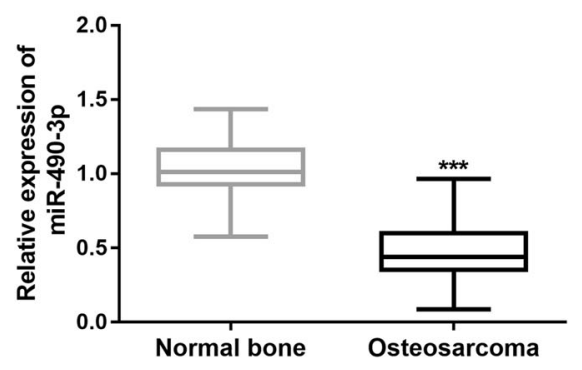

B
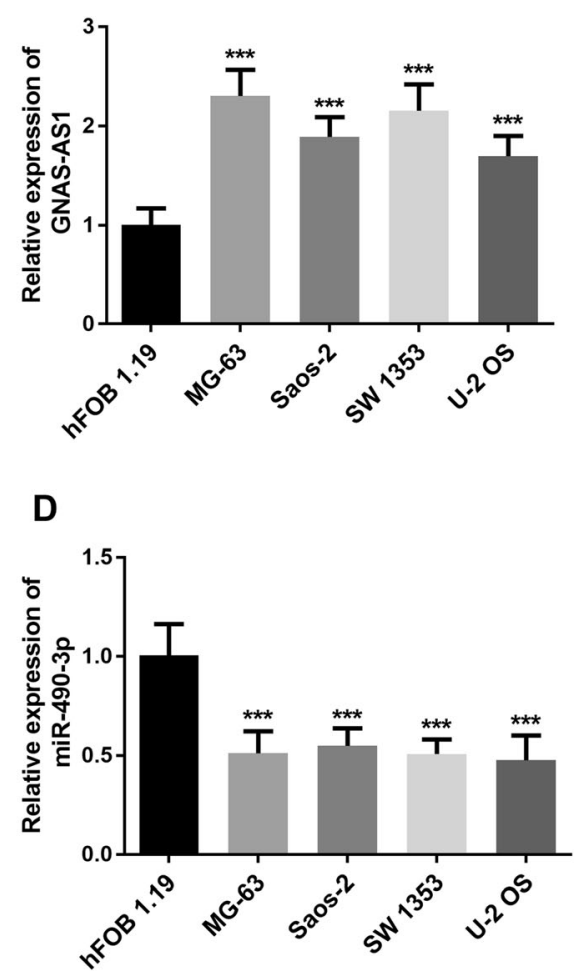

Fig. 1 LncRNA GNAS-AS1 is downregulated in osteosarcoma tissues and cell lines. A The relative expression of IncRNA GNAS-AS1 in osteosarcoma tissues and corresponding normal bone tissue samples $(n=108)$. B LncRNA GNAS-AS1 expression in osteosarcoma cell lines (MG63, Saos-2, SW 1353, U-2 OS) and one normal osteoblast cell line hFOB 1.19. C The relative expression of miR-490-3p in osteosarcoma tissues and corresponding normal bone tissue samples $(n=108)$. D miR-490-3p expression in osteosarcoma cell lines (MG-63, Saos-2, SW 1353, U-2 OS) and one normal osteoblast cell line hFOB 1.19. Data are all obtained from qRT-PCR. ${ }^{* * *} P<0.001$ 
Relationships between IncRNA GNAS-AS1 and clinicopathologic factors

The correlations between lncRNA GNAS-AS1 and clinicopathological variables of OS patients are presented in Table 1. According to the mean value of lncRNA GNAS-AS1 expression, the OS patients were divided into high expression group $(n=59)$ and low expression group $(n=49)$. From Table 1, overexpression of lncRNA GNAS-AS1 was significantly related to Enneking stage $(P=0.018)$ and distant metastasis $(P=0.030)$ in OS patients. While no significant differences were obtained between lncRNA GNAS-AS1 expression and other parameters (all $P>0.05)$.

\section{Increase of IncRNA GNAS-AS1 correlates with poor prognosis in OS patients}

The discrimination of overall survival between high and low lncRNA GNAS-AS1 expression group was assessed by Kaplan-Meier plots with $95 \%$ confidence intervals (log-rank test $P=0.017$, Fig. 2). High lncRNA GNASAS1 expression group showed shorter overall survival $(44.1 \pm 3.01)$ than low lncRNA GNAS-AS1 expression group $(54.0 \pm 2.5)$. Moreover, to determine the prognostic significance contributed by lncRNA GNAS-AS1 expression after accounting for other important prognostic factors, multivariate Cox proportional hazards regression

Table 1 Correlation between IncRNA GNAS-AS1 expression levels and clinical features in OS patients

\begin{tabular}{|c|c|c|c|c|}
\hline \multirow[t]{2}{*}{ Parameter } & \multirow{2}{*}{$\begin{array}{l}\text { Cases } \\
\text { no. } \\
(n= \\
108)\end{array}$} & \multicolumn{2}{|c|}{ LncRNA GNAS-AS1 expression } & \multirow[t]{2}{*}{$P$} \\
\hline & & Low $(n=49)$ & High $(n=59)$ & \\
\hline Gender & & & & 0.239 \\
\hline Male & 55 & 28 & 27 & \\
\hline Female & 53 & 21 & 32 & \\
\hline Age (years) & & & & 0.586 \\
\hline$<19$ & 52 & 25 & 27 & \\
\hline$\geq 19$ & 56 & 24 & 32 & \\
\hline Enneking stage & & & & $0.018^{*}$ \\
\hline$|-| \mid A$ & 44 & 26 & 18 & \\
\hline$\|B-1\|$ & 64 & 23 & 41 & \\
\hline Tumor size & & & & 0.069 \\
\hline$<8 \mathrm{~cm}$ & 58 & 31 & 27 & \\
\hline$\geq 8 \mathrm{~cm}$ & 50 & 18 & 32 & \\
\hline Location of lesion & & & & 0.957 \\
\hline Axial bone & 51 & 23 & 28 & \\
\hline Others & 57 & 26 & 31 & \\
\hline Distant metastasis & & & & $0.030^{*}$ \\
\hline Absence & 65 & 35 & 30 & \\
\hline Presence & 43 & 14 & 29 & \\
\hline
\end{tabular}

was used to assess the impact of various prognostic factors. As shown in Table 2, lncRNA GNAS-AS1 expression were identified as significant and independent prognostic variable $(\mathrm{HR}=4.519,95 \% \mathrm{CI}=1.473-13.865$, $P=0.008)$.

Knockdown of IncRNA GNAS-AS1 inhibited proliferation, invasion, and migration of human OS cells

To clarify the roles of lncRNA in tumorigenesis and the progression of OS, small interference RNA (si-GNAS-AS1) was used to knock down lncRNA GNAS-AS1 in MG-63 and SW 1353 cells. siRNA exhibited significant inhibition potency in these two cell lines $(P<0.001$, Fig. 3A). Correspondingly, we found an inhibition of cell proliferation with IncRNA GNAS-AS1 knockdown in these two OS cells $(P<$ 0.05, Fig. 3B, C). IncRNA GNAS-AS1 has also been reported to promote cell migration and invasion in ER+ breast cancer [18]; thus, we assayed for the effects of lncRNA GNAS-AS1 knockdown on cell migration and invasion in OS cells using transwell assay. The cell invasion and migration of MG-63 and SW 1353 cells with IncRNA GNAS-AS1 interference was suppressed $(P<0.05$, Fig. 3D, E).

\section{miR-490-3p was target miRNA of IncRNA GNAS-AS1}

LncBase predicted v.2 and lncRNASNP2 showed miR490-3p was one of the IncRNA GNAS-AS1 target miRNAs (Fig. 4A). Since miR-490-3p has shown a decreased expression in OS, and the expression level was strongly but negatively correlated with the expression of lncRNA GNAS-AS1 by Pearson's correlation coefficient $(r=$ $0.8829, P<0.001$, Fig. 4B). And inhibition of lncRNA GNAS-AS1 can lead an increase in the expression of miR-490-3p $(P<0.01$, Fig. 4C). To verify this further, luciferase assay was used to analyze the effect of miR-490$3 p$ mimic or inhibitor on the luciferase activity of WTGNAS-AS1 or MUT-GNAS-AS1. The luciferase activity of wide-type constructs exhibited significant reduction under miR-490-3p mimic and rise under miR-490-3p inhibitor $(P<0.001$, Fig. 4D). Whereas the luciferase activity of MUT-GNAS-AS1 has no alteration with the existence of miR-490-3p mimic or inhibitor.

Furtherly, a rescue experiment was performed. The transfection efficiency was confirmed by miR-490-3p expression $(P<0.001$, Fig. $5 \mathrm{~A})$. In the followed CCK8 assay, cell proliferation reduced by lncRNA GNAS-AS1 knockdown can be restored by inhibition of miR-490-3p $(P<0.05$, Fig. 5B, C). Furthermore, cell migration and invasion detected by transwell assay were increased again when si-GNAS-AS1 and miR-490-3p inhibitor coexisted $(P<0.05$, Fig. 5D, E). Rescue experiments suggest that miR-490-3p can reverse the cellular effect of lncRNA GNAS-AS1. 


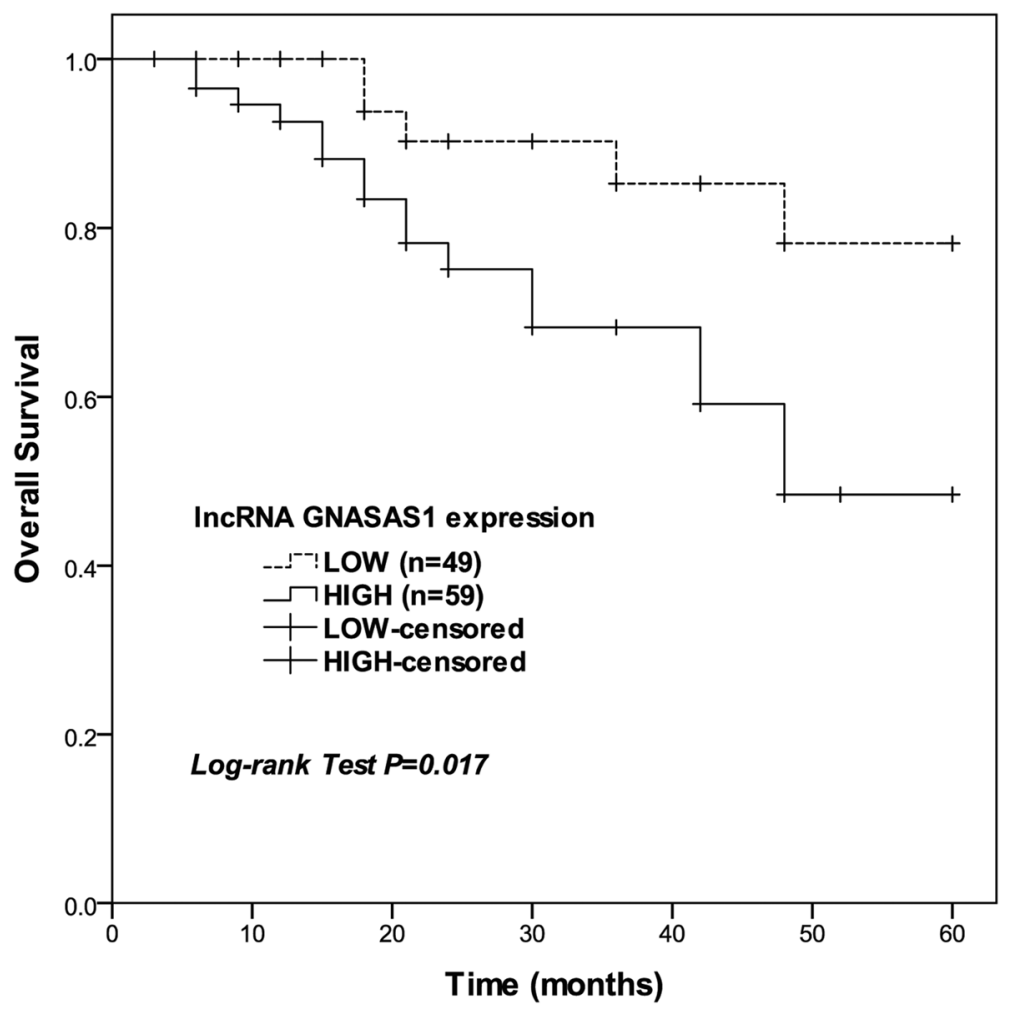

Fig. 2 Overall survival times were plotted via Kaplan-Meier survival curves and analyzed by log-rank tests (log-rank $P=0.017$ )

\section{Discussion}

Despite cancer is usually common in adults than children and adolescents [21], OS is characteristic of a bimodal age distribution with an adolescent $(0-20$ years old) and an elderly (centered at age 75 years) peak in incidence [22]. Considering the majority of children and adolescents involved in high-grade OS whose remaining life expectancy is crucial, the prediction of prognosis becomes very important [23]. Besides, the prognosis for patients with metastatic OS is much poorer than for those with only primary OS [24]. Therefore, new prognosis predictor should be developed to improve the outcome estimate.

Table 2 Multivariate Cox analysis of prognostic factors in OS patients based on the overall survival

\begin{tabular}{llll}
\hline Parameter & \multicolumn{3}{l}{ Multivariate analysis } \\
\cline { 2 - 4 } & HR & $\mathbf{9 5 \% C l}$ & $\boldsymbol{P}$ \\
\hline LncRNA GNAS-AS1 & 4.519 & $1.473-13.865$ & 0.008 \\
Gender & 2.025 & $0.732-5.605$ & 0.174 \\
Age & 1.167 & $0.473-2.881$ & 0.737 \\
Tumor size & 1.671 & $0.591-4.726$ & 0.333 \\
Location of lesion & 2.371 & $0.875-6.419$ & 0.089 \\
Enneking stage & 8.172 & $0.973-68.664$ & 0.053 \\
Distant metastasis & 5.291 & $1.455-19.242$ & 0.011 \\
\hline
\end{tabular}

The prognosis value of lncRNAs has been gradually revealed recently, such as lnc-HOST2, lncRNA CBR3-AS1, or lncRNA TP73-AS1 [25-27]. Since lncRNA GNASAS1 has been characterized as one lncRNA significantly associated with OS patients' survival by bioinformatics, this study focused on the clinical significance of lncRNA GNAS-AS1 in the OS cases of our institution. Firstly, the expression of IncRNA GNAS-AS1 in 108 OS tissues and a panel of cell lines were detected by qRT-PCR, and was found to be upregulated in tumor tissues and cancerous cell lines. In line with this, upregulation of lncRNA GNAS-AS1 was also found in nasopharyngeal cancer, breast cancer, and non-small cell lung cancer [17-19]. Furtherly, the patients were divided into two groups based on the mean expression value of lncRNA GNAS-AS1, and the association between the expression and clinical parameters was accessed by chi-square test, which showed the high expression of lncRNA GNASAS1 was related to high Enneking stage and positive distant metastasis. This relationship suggests to us the potential prognosis value of lncRNA GNAS-AS1. In order to verify the prognosis value, Kaplan-Meier curves were plotted to directly display the distinction of the overall survival between patients with high lncRNA GNAS-AS1 expression and low expression, and multivariate Cox analysis was introduced to access the statistical significance of IncRNA GNAS-AS1 as prognostic factor. The 

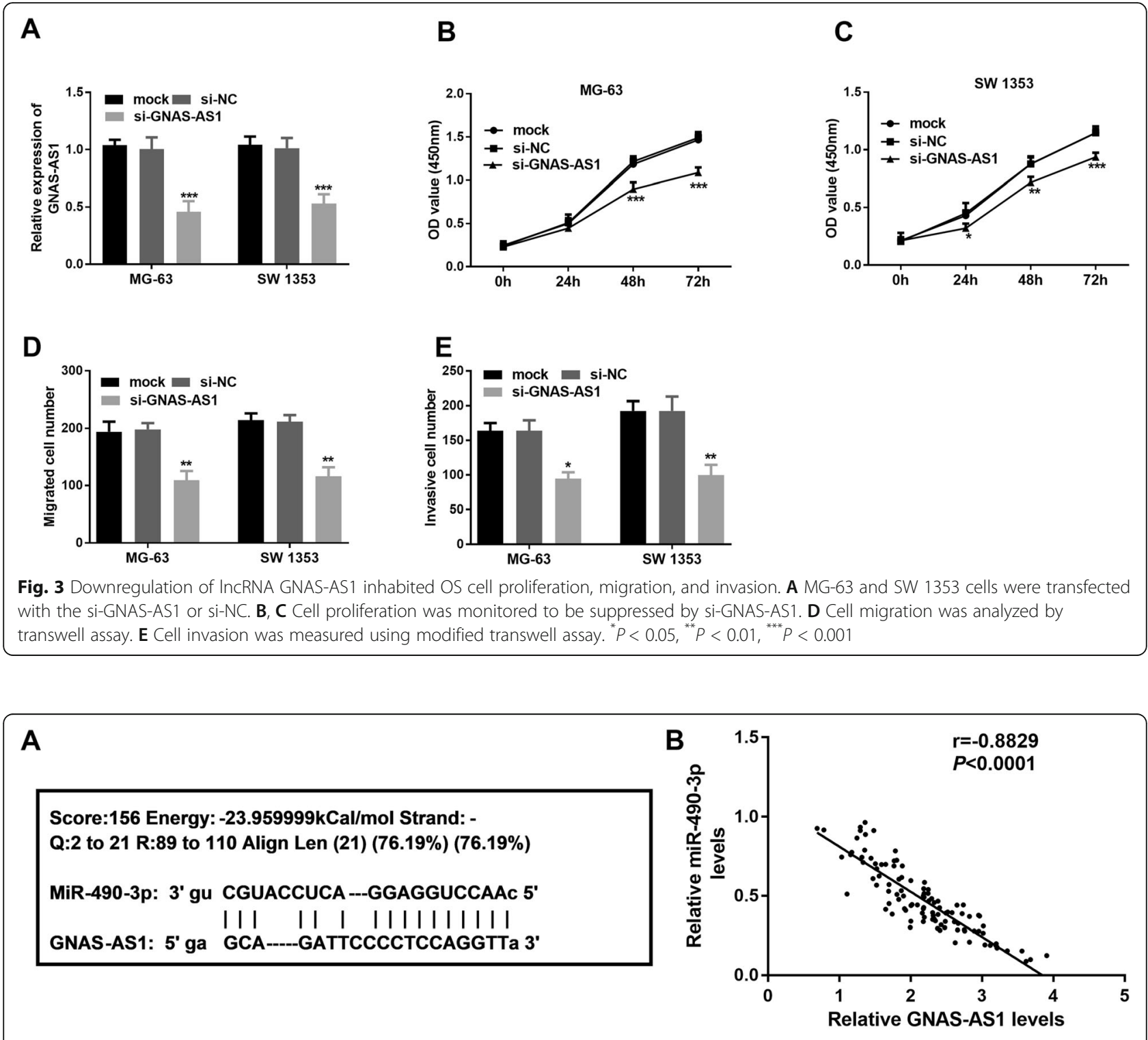

C
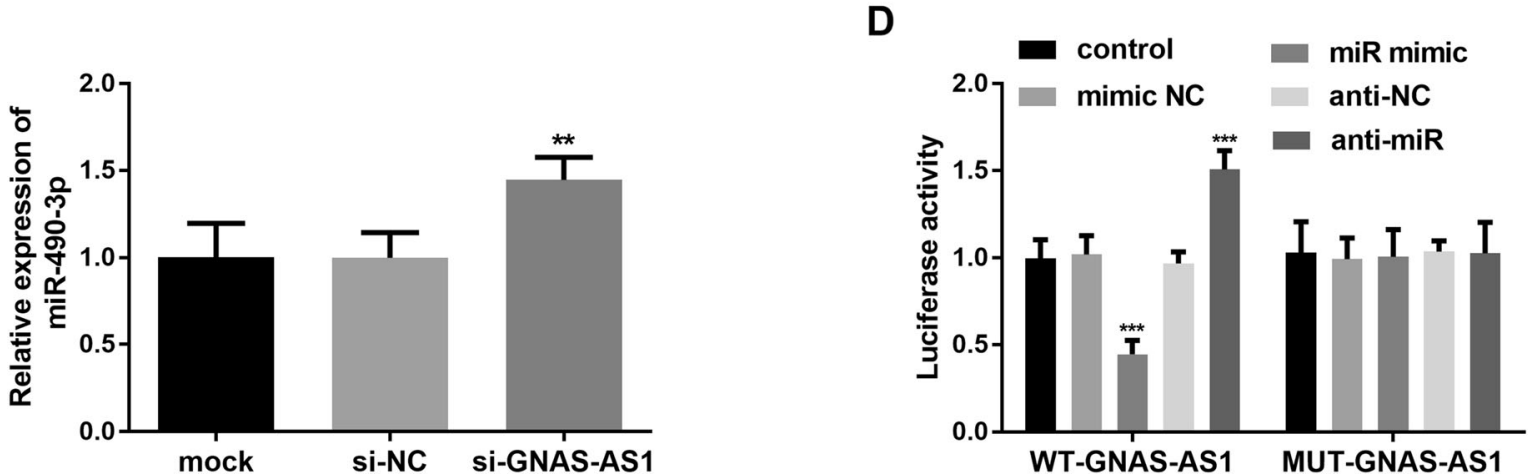

Fig. 4 miR-490-3p was downstream miRNA of IncRNA GNAS-AS1. A Binding sites for IncRNA GNAS-AS1 in the miR-490-3p 3'UTR were predicted using IncRNASNP2. B Expression of IncRNA GNAS-AS1 and miR-490-3p is negatively correlated. C Expression of miR-490-3p was raised when IncRNA GNAS-AS1 was inhibited. D pLHCX retroviral vector with wild-type IncRNA GNAS-AS1 (WT-GNAS-AS1) and mutated one (MUT-GNAS-AS1) were constructed, and co-transfected into MG-63 cells with the miR-490-3p mimic or inhibitor. The relative luciferase activity was measured. ${ }^{* *} P<$ $0.01,{ }^{* * *} P<0.001$ 


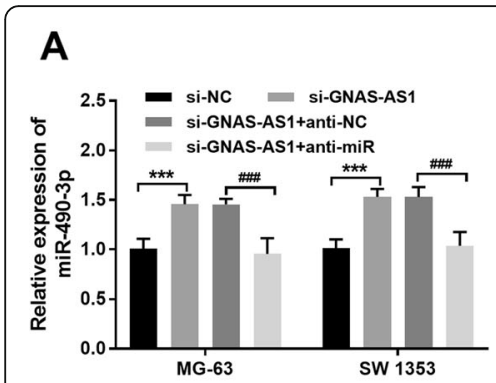

D

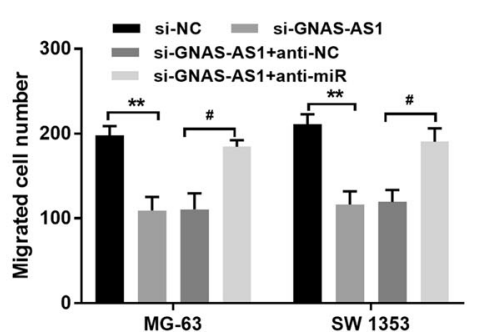

B

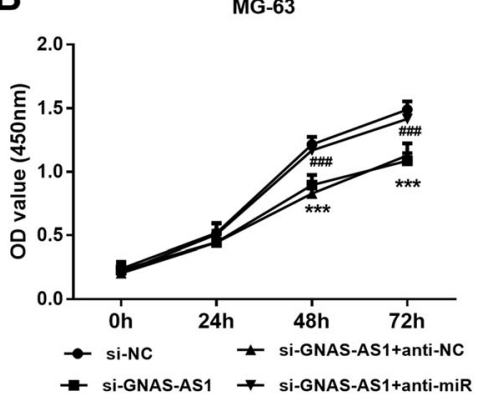

$\mathbf{E}$

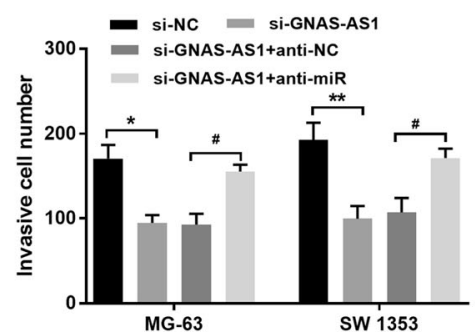

Fig. 5 miR-490-3p counteracted the cellular function of IncRNA GNAS-AS1. A The effect of transfection was confirmed by qRT-PCR. B, C miR-490$3 p$ downregulation can offset the suppression of proliferation by si-GNAS-AS1. $\mathbf{D}$ miR-490-3p downregulation can neutralize the inhibition of migration by si-GNAS-AS1. E miR-490-3p downregulation can abolish the repression of cell invasion by si-GNAS-AS1. ${ }^{*} P<0.05,{ }^{* *} P<0.01,{ }^{* * *} P<$

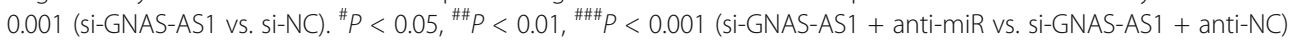

result showed lncRNA GNAS-AS1 was identified as an independent prognosis factor for overall survival.

Elucidation of the molecular basis on which lncRNAs affect OS development and progression is essential to establish better treatment options [1, 28]. Recently, lncRNAs have been identified that participate in OS progression, including cancerous cell growth, metastasis, and apoptosis [29-31]. LncRNA GNAS-AS1 was an important gene related to the formation and progression of nasopharyngeal carcinoma and breast cancer $[17,18]$. In this study, the effect of IncRNA GNAS-AS1 interference on OS cellular growth and metastasis was explored. By CCK-8 assay, the proliferation was found to be suppressed by IncRNA GNAS-AS1 knockdown, and by transwell assay, the migration and invasion of OS cells were inhibited by IncRNA GNAS-AS1 downregulation. These suggest that lncRNA GNAS-AS1 plays a role in promoting OS development, which can also be a new potential therapeutic target for OS.

LncRNAs can act as microRNA sponge to modulate gene expression, which involves various ncRNAs in interconnected competitive regulatory interactions, known as competing endogenous RNA (ceRNA) network [32]. Thus, the aberrant expression of IncRNA could influence the downstream miRNA directly, culminating in development and progression of cancer. To find the possible target miRNAs of lncRNA GNAS-AS1, LncBase
Predicted v.2 and lncRNASNP2 were used to search, and miR-490-3p got our attention since its decreased expression and prognosis value in OS [33]. To confirm the binding relationship of miR-490-3p and lncRNA GNASAS1, the expression of miR-490-3p under lncRNA GNAS-AS1 interference was detected to be increased, and the expression profile of these two RNA was negatively correlated. Luciferase reporter assay and rescue experiment furtherly confirmed lncRNA GNAS-AS1 act as ceRNA of miR-490-3p to exert its cellular function.

The limitation of study population just in our single organization is disadvantage of this study. A larger number of subjects would be needed to validate lncRNA GNAS-AS1 values in OS prognosis. Moreover, in vivo experiments related to the cellular function of lncRNA GNAS-AS1 are needed to explore the deep mechanism. Future research would benefit from evaluating lncRNA GNAS-AS1 clinical significance in larger samples. In addition, it would be useful for development of new treatment tool to explore the thorough mechanism of lncRNA GNAS-AS1 effecting on the cellular function.

\section{Conclusion}

In conclusion, lncRNA GNAS-AS1 was with an increased expression in OS, and this overexpression was associated with unfavorable pathological characteristics and shorter overall survival. Knockdown of lncRNA 
GNAS-AS1 inhibits cell growth, migration, and invasion. LncRNA GNAS-AS1 has the potential to be used as an independent prognostic factor and new molecular therapeutic approach. Finally, further studies are desirable to validate the accuracy of lncRNA GNAS-AS1 as prognosis biomarker in a series of patient studies in order to determine the accuracy in a clinical setting.

\section{Acknowledgements}

Not applicable.

\section{Authors' contributions}

All authors developed the original idea, analyzed data, and wrote the manuscript. ZM and YD performed all the experiments. ZM and ZW contributed to the acquisition of patients and tissue specimens. All authors read and approved the final manuscript.

\section{Funding}

Not applicable.

\section{Availability of data and materials}

Corresponding authors may provide data and materials.

\section{Declarations}

\section{Ethics approval and consent to participate}

The research ethics committee of General Hospital of Ningxia Medical University approved the study. All patients signed a consent form before surgery, agreeing to use their tissues for this study.

\section{Consent for publication}

All patients signed written consent form for publication.

\section{Competing interests}

The authors declare that they have no competing interests.

\begin{abstract}
Author details
${ }^{1}$ Department of Trauma Orthopedics, General Hospital of Ningxia Medical University, 804 South Shengli Street, Yinchuan, Ningxia 750004, People's Republic of China. ${ }^{2}$ Operating Room, General Hospital of Ningxia Medical University, Yinchuan, Ningxia 750004, People's Republic of China. ${ }^{3}$ Department of Orthopedics, Rizhao Central Hospital, Rizhao, Shandong 276000, People's Republic of China.
\end{abstract}

Received: 3 June 2021 Accepted: 12 July 2021

Published online: 28 July 2021

\section{References}

1. Huynh NP, Anderson BA, Guilak F, McAlinden A. Emerging roles for long noncoding RNAs in skeletal biology and disease. Connect Tissue Res. 2017; 58(1):116-41. https://doi.org/10.1080/03008207.2016.1194406.

2. Simpson E, Brown HL. Understanding osteosarcomas. JAAPA. 2018;31(8):159. https://doi.org/10.1097/01.JAA.0000541477.24116.8d.

3. Anderson ME. Update on survival in osteosarcoma. Orthop Clin North Am. 2016;47(1):283-92. https://doi.org/10.1016/j.ocl.2015.08.022.

4. Mediouni M, Schlatterer DR. Orthopaedic tumors: what problems are we solving, and are universities and major medical centers doing enough? J Orthop. 2017;14(2):319-21. https://doi.org/10.1016/j.jor.2017.03.014.

5. Spraker-Perlman HL, Barkauskas DA, Krailo MD, Meyers PA, Schwartz CL, Doski J, et al. Factors influencing survival after recurrence in osteosarcoma: a report from the children's oncology group. Pediatr Blood Cancer. 2019;66(1): e27444. https://doi.org/10.1002/pbc.27444

6. Walia MK, Castillo-Tandazo W, Mutsaers AJ, Martin TJ, Walkley CR. Murine models of osteosarcoma: a piece of the translational puzzle. J Cell Biochem. 2018;119(6):4241-50. https://doi.org/10.1002/jcb.26601.

7. Mediouni M, Schlatterer DR, Madry H, Cucchiarini M, Rai B. A review of translational medicine. The future paradigm: how can we connect the orthopedic dots better? Curr Med Res Opin. 2018;34:1217-29.
8. Liu ZB, Wang JA, Lv RQ. Downregulation of long non-coding RNA DBH-AS1 inhibits osteosarcoma progression by PI3K-AKT signaling pathways and indicates good prognosis. Eur Rev Med Pharmacol Sci. 2019;23(4):1418-27. https://doi.org/10.26355/eurrev_201902_17098.

9. He W, Lu M, Xiao D. LSINCT5 predicts unfavorable prognosis and exerts oncogenic function in osteosarcoma. Biosci Rep. 2019;39(5). https://doi. org/10.1042/BSR20190612.

10. Teng J, Guo X, Wang H. CCEPR is a novel clinical biomarker for prognosis and regulates cell proliferation through PCNA in osteosarcoma. J Cell Biochem. 2019;120(8):12796-802. https://doi.org/10.1002/jcb.28550.

11. Zha Z, Han Q, Liu W, Huo S. IncRNA GAS8-AS1 downregulates IncRNA UCA1 to inhibit osteosarcoma cell migration and invasion. J Orthop Surg Res. 2020;15:38.

12. Anastasiadou E, Jacob LS, Slack FJ. Non-coding RNA networks in cancer. Nat Rev Cancer. 2018;18(1):5-18. https://doi.org/10.1038/nrc.2017.99.

13. Zheng S, Jiang F, Ge D, Tang J, Chen H, Yang J, et al. LncRNA SNHG3/ miRNA-151a-3p/RAB22A axis regulates invasion and migration of osteosarcoma. Biomed Pharmacother. 2019;112:108695. https://doi.org/10.1 016/j.biopha.2019.108695.

14. Huang Q, Shi SY, Ji HB, Xing SX. LncRNA BE503655 inhibits osteosarcoma cell proliferation, invasion/migration via Wnt/B-catenin pathway. Biosci Rep. 2019;39.

15. Fu D, Lu C, Qu X, Li P, Chen K, Shan L, et al. LncRNA TTN-AS1 regulates osteosarcoma cell apoptosis and drug resistance via the miR-134-5p/MBTD1 axis. Aging (Albany NY). 2019;11(19):8374-85. https://doi.org/10.18632/a ging. 102325.

16. Zhang W, Ren X, Qi L, Zhang C, Tu C, Li Z. The value of IncRNAs as prognostic biomarkers on clinical outcomes in osteosarcoma: a meta-analysis. BMC Cancer. 2021;21(1):202. https://doi.org/10.1186/s12885-021-07882-w.

17. Wang $X Q, X u H$, Wang $\mathrm{CH}$, Xie $H$. Long non-coding RNA GNAS-AS1 promotes cell migration and invasion via regulating $W n t / \beta$-catenin pathway in nasopharyngeal carcinoma. Eur Rev Med Pharmacol Sci. 2020;24(6):307784. https://doi.org/10.26355/eurrev_202003_20672.

18. Liu SQ, Zhou ZY, Dong X, Guo L, Zhang KJ. LncRNA GNAS-AS1 facilitates ER+ breast cancer cells progression by promoting M2 macrophage polarization via regulating miR-433-3p/GATA3 axis. Biosci Rep. 2020;40.

19. Li Z, Feng C, Guo J, Hu X, Xie D. GNAS-AS1/miR-4319/NECAB3 axis promotes migration and invasion of non-small cell lung cancer cells by altering macrophage polarization. Funct Integr Genomics. 2020;20(1):17-28. https://doi.org/10.1007/s10142-019-00696-x.

20. Gao H, Guo Y, Zhang M, Yi Z. Comprehensive characterization of prognostic long noncoding RNAs in osteosarcoma. Biomed Res Int. 2020;2020:6725753.

21. Torre LA, Siegel RL, Ward EM, Jemal A. Global cancer incidence and mortality rates and trends--an update. Cancer Epidemiol Biomark Prev. 2016; 25(1):16-27. https://doi.org/10.1158/1055-9965.EPI-15-0578.

22. Moore DD, Luu HH. Osteosarcoma. Cancer Treat Res. 2014;162:65-92. https://doi.org/10.1007/978-3-319-07323-1_4.

23. Zhang H, Su X, Guo L, Zhong L, Li W, Yue Z, et al. Silencing SATB1 inhibits the malignant phenotype and increases sensitivity of human osteosarcoma U2OS cells to arsenic trioxide. Int J Med Sci. 2014;11(12):1262-9. https://doi. org/10.7150/ijms.10038.

24. Song K, Song J, Lin K, Chen F, Ma X, Jiang J, et al. Survival analysis of patients with metastatic osteosarcoma: a surveillance, epidemiology, and end results population-based study. Int Orthop. 2019;43(8):1983-91. https:// doi.org/10.1007/s00264-019-04348-4.

25. Qi XD, Xu SY, Song Y. Prognostic value of long non-coding RNA HOST2 expression and its tumor-promotive function in human osteosarcoma. Eur Rev Med Pharmacol Sci. 2018;22(4):921-7. https://doi.org/10.26355/eurrev_2 01802 14371.

26. Zhang Y, Meng W, Cui H. LncRNA CBR3-AS1 predicts unfavorable prognosis and promotes tumorigenesis in osteosarcoma. Biomed Pharmacother. 2018; 102:169-74. https://doi.org/10.1016/j.biopha.2018.02.081.

27. Chen $X$, Zhou Y, Liu S, Zhang D, Yang X, Zhou Q, et al. LncRNA TP73-AS1 predicts poor prognosis and functions as oncogenic IncRNA in osteosarcoma. J Cell Biochem. 2018.

28. Rickel K, Fang F, Tao J. Molecular genetics of osteosarcoma. Bone. 2017;102: 69-79. https://doi.org/10.1016/j.bone.2016.10.017.

29. Lin H, Wu T, Peng L, Su W, Wang Y, Li X, et al. Lnc-MAP6-1:3 knockdown inhibits osteosarcoma progression by modulating Bax/Bcl-2 and Wnt/ $\beta$ catenin pathways. Int J Med Sci. 2020;17(15):2248-56. https://doi.org/10.71 50/ijms.47405. 
30. Yu X, Pang L, Yang T, Liu P. IncRNA LINC01296 regulates the proliferation, metastasis and cell cycle of osteosarcoma through cyclin D1. Oncol Rep. 2018;40(5):2507-14. https://doi.org/10.3892/or.2018.6674.

31. Yang W, Shan Z, Zhou X, Peng L, Zhi C, Chai J, et al. Knockdown of IncRNA GHET1 inhibits osteosarcoma cells proliferation, invasion, migration and EMT in vitro and in vivo. Cancer Biomark. 2018;23(4):589-601. https://doi.org/1 $0.3233 / \mathrm{CBM}-181863$

32. Chan JJ, Tay Y. Noncoding RNA:RNA regulatory networks in cancer. Int J Mol Sci. 2018;19.

33. Tang B, Liu C, Zhang QM, Ni M. Decreased expression of miR-490-3p in osteosarcoma and its clinical significance. Eur Rev Med Pharmacol Sci. 2017; 21(2):246-51.

\section{Publisher's Note}

Springer Nature remains neutral with regard to jurisdictional claims in published maps and institutional affiliations.

Ready to submit your research? Choose BMC and benefit from:

- fast, convenient online submission

- thorough peer review by experienced researchers in your field

- rapid publication on acceptance

- support for research data, including large and complex data types

- gold Open Access which fosters wider collaboration and increased citations

- maximum visibility for your research: over $100 \mathrm{M}$ website views per year

At $\mathrm{BMC}$, research is always in progress.

Learn more biomedcentral.com/submissions 